\title{
Practical management of pleural empyema
}

\author{
G.F. Tassi1, G.P. Marchetti1, V. Pinelli1, S. Chiari2
}

ABSTRACT: Practical management of pleural empyema. G.F. Tassi, G.P. Marchetti, V. Pinelli, S. Chiari.

Empyema is defined as pus in the thoracic cavity due to pleural space infection and has a multifactorial underlying cause, although the majority of cases are post-bacterial pneumonia. Despite treatment with antibiotics, patients with empyema have a considerable morbidity and mortality due at least in part to inappropriate management of the effusion.

Timely diagnosis of pleural space infection and rapid initiation of effective pleural drainage represent fundamental principles for managing patients with empyema.

Ultrasound is particularly useful to identify early fibrin membranes and septations in the pleural cavity conditioning treatment strategy.
Empyema and large or loculated effusion with a $\mathrm{pH}<7.20$ need to be drained.

Thoracoscopy has largely been used in pleural effusion due to lung infection.

Whereas the efficacy of video-assisted thoracic surgery (VATS) in empyema management has been evaluated in several retrospective studies showing favourable results, less is known about the role of medical thoracoscopy (MT) in pleural infection. MT, appears to be safe and successful in multiloculated empyema treatment. It is also lower in cost and in frail patients is better tolerated than VATS which requires tracheal intubation.

Monaldi Arch Chest Dis 2010; 73: 3, 124-129.

Keywords: Empyema, Parapneumonic pleural effusion, Chest drainage, Medical thoracoscopy, Video-assisted thoracoscopic surgery.

1 Divisione di Pneumologia, Spedali Civili di Brescia, Brescia,

2 Scuola di Specializzazione in Malattie dell'Apparato Respiratorio, Università degli Studi di Brescia, Italy.

Correspondence: Dr.ssa Valentina Pinelli, Division of Pneumology, Spedali Civili Brescia, Piazzale Spedali Civili 1, 25103 Brescia, Italy; e-mail: valentina.pinelli@gmail.com

\section{Introduction}

Pleural empyema, by definition the presence of pus in the pleural space (not an effusion with organisms seen on Gram stain or low-pH pleural effusion, as indicated in some studies [1]), is still associated with significant morbidity and mortality in adults and children.

It was first described in the $5^{\text {th }}$ century B.C. by Hippocrates in his aphorisms: "pleuritis that does not clear up in fourteen days results in empyema". Hippocrates proposed drainage as a treatment for it [2] and for hundreds of years empyema was treated using open drainage.

Today the treatment of empyema can be summarised as appropriate antibiotic therapy combined with medical or surgical pleural space drainage. Traditionally, the management of empyema has been empirical. Solid evidence based on controlled clinical trial data to guide practice is still lacking, but recent studies are leading to more focused management guidelines.

\section{Epidemiology}

Pleural infection affects 65,000 individuals in the United States and United Kingdom each year and carries a mortality of approximately $15 \%$ [3-5]; moreover some recent North American studies have demonstrated an increasing rate of empyema with a rise in incidence of $2.8 \%$ per year [5] and $12.4 \%$ in 2003 compared to 1995 [6]. Very young or elderly people are more commonly afflicted with it.

Risk factors for development include diabetes mellitus, alcohol abuse, poor dental hygiene, gastro-oesophageal reflux, rheumatoid arthritis, chronic lung disease, and intravenous drug abuse. Approximately one third of cases occur in the absence of any identifiable risk factors [5], suggesting that variation in bacterial virulence or host immune defence may also play important role in empyema development.

Empyema may develop as a complication of pneumonia, or may follow surgery, trauma, iatrogenic procedures, or, rarely, bronchial obstruction from a tumour or foreign body. Pleural infection may also occur as a "primary" infection, without evidence of lung parenchymal infection.

\section{Pathophysiologic classification and clinical staging of pleural infection}

Pleural effusion develops when the balance of pleural fluid formation and removal is altered. 
Pleural effusion secondary to pneumonia is termed parapneumonic effusion. Most of these effusions remain sterile and are resolved with antibiotic therapy (uncomplicated parapneumonic effusion), but infections of the pleural space develop in a small subset of patients and require drainage for full recovery (complicated parapneumonic effusion). Without effective drainage, complicated parapneumonic, effusion progresses to frank intrapleural pus (empyema) [7].

In 2000 a panel convened by the American College of Chest Physicians proposed guidelines on the medical and surgical treatment of parapneumonic effusions, and produced an annotated table for evaluating the risk for poor outcome.

Three variables - pleural space anatomy, pleural fluid bacteriology, and pleural fluid chemistry - were used in this annotated table to categorise patients into four separate risk levels for poor outcome: category 1 (very low risk), 2 (low risk), 3 (moderate risk), and 4 (high risk). Category 4 was characterised by the presence of pus in the pleural cavity, therefore including "true" empyemas (Table 1) [8].

According to Light, empyema is usually classified as simple empyema (category 6) when there is the presence of frank pus, single locule or free flowing effusion or as complex empyema (category 7), when there is frank pus with multiple loculation [9].

Another classification is the 1962 ATS classification [10] based on the natural history of the disease, and still employed for its usefulness from the clinical point of view. Empyema was subdivided into 3 stages or phases: exudative, fibro-purulent, and organised. The acute or exudative phase lasts for several days and is characterised by an effusion that is free-moving in the pleural cavity. Progression to the fibro-purulent phase is characterised by a reduced endocavitary fibrinolysis which causes fibrin deposition on the pleural surfaces and by a fluid which is cloudy and viscous.

The chronic or organised phase follows on from the fibrin and collagen deposition which creates a fibrous thickening of the pleura, a sort of 'peel' which traps the lung. This differentiation into stages is of course a simplification of a biological process which evolves progressively, but it is useful in practice because the therapeutic approach is very different in each stage.

\section{Diagnosis}

\section{Imaging}

Imaging techniques play a key role in the management of empyema. The chest X-ray, frontal and lateral view, maintains its basic role for establishing the presence and amount of pleural effusion and for follow-up of the patients.

However, the routine use of thoracic ultrasound (US) in patients with suspected pleural infection should be encouraged. US is particularly helpful in determining the nature of localised or diffuse pleural opacities, and is more sensitive than X-ray in identifying small or loculated pleural effusion [11].

Classically, no clinical or radiological findings unequivocally identify a complicated pleural effusion requiring drainage from an uncomplicated one that might be treated with conservative management. It would be a major advance if non-invasive tests were able to identify those patients who need pleural space drainage.

Some preliminary studies do identify ultrasound findings such as multiple loculations (septation) and reduction of mobility of the diaphragm correlating with prognosis [12-14].

Ultrasonography is an easy, accessible, economical and helpful tool to identify and quantify pleural septation at an early stage (figure 1). With pleural ultrasonography, the pleural shadowing seen on chest radiography or contrast enhanced thoracic CT can be examined in greater detail. Liquid loculation can be distinguished from solid parts and septations. Another important feature is the evaluation of diaphragmatic motility: immobilisation of the diaphragm can be frequently seen in heavily septated empyemas or fibrothorax.

Table 1. - Categorizing risk for poor outcome in patients with parapneumonic effusion and empyema

\begin{tabular}{|c|c|c|c|c|c|c|c|}
\hline Pleural Space Anatomy & & $\begin{array}{l}\text { Pleural fluid } \\
\text { Bacteriology }\end{array}$ & & $\begin{array}{l}\text { Pleural Fluid } \\
\text { Chemistry }\end{array}$ & Category & $\begin{array}{l}\text { Risk of poor } \\
\text { Outcome }\end{array}$ & Drainage \\
\hline $\begin{array}{l}\mathrm{A}_{0} \text { : Minimal, free-flowing } \\
\text { effusion }(<10 \mathrm{~mm} \\
\text { on lateral decubitus })\end{array}$ & and & $\begin{array}{l}\mathrm{B}_{\mathrm{x}} \text { : culture } \\
\text { Gram stain } \\
\text { results unknown }\end{array}$ & and & $\mathrm{C}_{\mathrm{x}}: \mathrm{pH}$ unknown & 1 & Very low & No \\
\hline $\begin{array}{l}\mathrm{A}_{1}: \text { Small to moderate } \\
\text { free-flowing effusion } \\
(>10 \mathrm{~mm} \text { and }<\text { one-half } \\
\text { hemithorax }\end{array}$ & and & $\begin{array}{l}\mathrm{B}_{0}: \text { negative } \\
\text { culture and } \\
\text { Gram stain }\end{array}$ & and & $\mathrm{C}_{0}: \mathrm{pH} \geq 7.20$ & 2 & Low & No \\
\hline \multirow{2}{*}{$\begin{array}{l}\mathrm{A}_{2} \text { : Large, free-flowing } \\
\text { effusion ( } \geq \text { one-half } \\
\text { hemithorax) loculated } \\
\text { effusion, or effusion with } \\
\text { thickened parietal pleura }\end{array}$} & or & $\begin{array}{l}\mathrm{B}_{1}: \text { postive } \\
\text { culture and } \\
\text { Gram stain }\end{array}$ & or & $\mathrm{C}_{1}: \mathrm{Ph}<7.20$ & 3 & Moderate & Yes \\
\hline & & $\mathrm{B}_{2}:$ pus & & & 4 & High & Yes \\
\hline
\end{tabular}



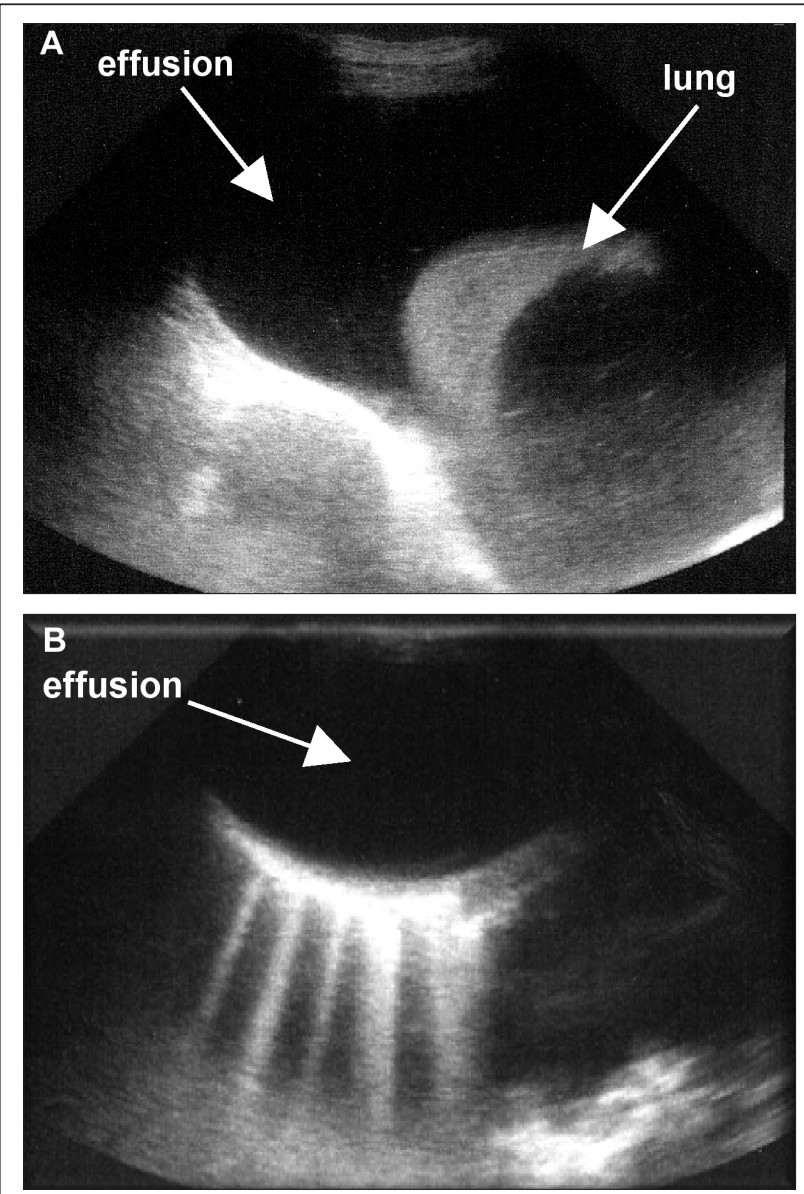

\section{Thoracentesis and pleural fluid analysis}

Diagnostic thoracentesis is crucial to demonstrate the presence of pus which identifies the empyema and it is important to remember that all patients with a pleural effusion in association with sepsis or a pneumonic illness require diagnostic pleural fluid sampling [7].

Biochemical and microbiological investigation of pleural fluid remains a complex subject. Despite a plethora of novel approaches in biochemical analysis, pleural fluid $\mathrm{pH}$ using a blood-gas machine remains an important assay to identify those effusions (i.e. those with $\mathrm{pH} \leq 7.2$ ) that may require chest tube drainage or thoracoscopy [16-17].

Similarly, there are limitations on pleural fluid microbiologial investigation. Jimènez et al. showed that the yield of pleural fluid culture in suspected cases of empyema is low and this is not due solely to antibiotic use prior to thoracentesis; pleural fluid appears to be a relatively poor sample from a microbiologial point of view. However, continuing to take pleural fluid cultures can probably be justified by the small subset of patients in whom an unusual or resistant organism is identified [18].

\section{Treatment}

Treatment, as a rule, is based on antibiotic therapy and complete drainage of the liquid to allow total lung re-expansion. The choice of treatment in individual cases is more often guided by available resources and the philosophy of physicians, rather than scientific data, and varies considerably between different hospitals, regions, and countries.

\section{Antibiotics}

Whilst in general the selection of antibiotics should be based on the results of blood and pleural fluid cultures, given the low rate of positive results of bacteriology ( $30 \%)$ in empyema antibiotic therapy always starts as empirical, frequently continues empirically and only in a minority of cases should be adapted to the laboratory results.

Aminopenicillins, penicillins combined with $\beta$-lactamase inhibitors (e.g. co-amoxiclav or piperacillin-tazobactam) and cephalosporins show good penetration of the pleural space.

Aminoglycosides should be avoided as they have poor penetration into the pleural space and may be inactive in the presence of pleural fluid acidosis.

Macrolide antibiotics are not indicated unless there is objective evidence or high clinical index of atypical pathogens.

In community-acquired empyema, treatment with an aminopenicillin (e.g. amoxicillin) will cover organisms such as $S$ pneumonia and $\mathrm{H}$ influenza, but a $\beta$-lactamase inhibitors as co-amoxiclav or metronidazole should also be given because of the frequent co-existence of penicillin-re- 
sistant aerobes including $S$ aureus and anaerobic bacteria. Clindamycin achieves a good penetration of the infected pleural space and can be used either alone or in combination with a cephalosporin.

In hospital-acquired empyema, usually secondary to a nosocomial pneumonia, antibiotics should be the therapy of choice to treat Gram-positive and Gram-negative aerobes and anaerobic organisms.

Recent studies have shown that there is a significant increase in MRSA infection causing hospital-acquired empyema, so empirical antibiotics in this case should initially include cover for MRSA until microbiological results are available.

Intravenous administration of antibiotics is often appropriate initially but can be changed to oral when objective clinical and biochemical improvement has been observed. The duration of treatment for pleural infection has not been assessed in detailed clinical trials, however antibiotics are often continued for at least 3 weeks and their suspension should again be based on clinical (e.g. improvement in temperature), biochemical (e.g. CRP), and radiological response [7].

\section{Chest tube drainage}

Lack of response to antibiotics, demonstrated by lack of clinical and radiological improvement, is a strong indication for chest tube drainage. The optimum size of chest tube and duration of drainage is still under discussion.

In parapneumonic effusion, small-bore drains $(\leq 14 \mathrm{Fr})$ are easier to insert, more comfortable and adequate for the drainage of infected pleural collections. But this is not the case with the presence of frank pus, where larger tubes $(\geq 24 \mathrm{Fr})$ are needed [19].

The first large multicentre study on chest drain diameter in the treatment of pleural infection has recently been published. The authors found no difference between small ( $\leq 10 \mathrm{Fr})$ and large ( $\geq 20 \mathrm{Fr})$ diameter drains for any impairment in clinical outcome except insertion discomfort [20].

Another developing area is the use of longterm catheters, particularly for patients with loculated empyema that are unsuitable for more aggressive treatment [21].

Removal of the chest drain is appropriate depending on two factors: first, after radiological confirmation of successful pleural drainage, i.e. reduction in the size of pleural collection on the chest $\mathrm{x}$-ray or thoracic ultrasound; and second, objective evidence of sepsis resolution, i.e. improvement in temperature and clinical condition and decreasing inflammatory markers (e.g. CRP). In-patient observation for 24 hours after drain removal is usual, although a longer period of rehabilitation may be necessary, as most patients will have been unwell and in hospital for a prolonged period [7].

\section{Failure of chest tube drainage}

The presence of loculations, which are characteristic of complicated parapneumonic effusion and fibrinopurulent empyema, frequently make the effusion resistant to drainage with a single chest tube. In these situations, either intrapleural fibrinolytics, medical thoracoscopy, video-assisted thoracic surgery (VATS) or, in advanced cases of chronic fibrotic empyema, open surgical decortication is used.

\section{Intrapleural fibrinolytics}

The role of intrapleural fibrinolytics remains under discussion. Until the Multicentre Intrapleural Sepsis Trial (MIST) was published, there was a broad consensus that their use was beneficial, although the existing data did not provide convincing evidence to support this.

Maskell et al showed in MIST that in an unselected group of empyema patients, intrapleural fibrinolysis through chest drains had no effect on outcomes such as mortality and admission length [22]. However, a more recent study published by Misthos et al that compares tube thoracostomy alone with tube thoracostomy and fibrinolysis, showed that the addition of fibrinolysis decreases the rate of surgical interventions (VATS or open decortication), and the length of hospital stay [23]. Similar conclusions have been reached in a recent review performed by the Cochrane Collaboration. The reasons for this difference are unclear, but the MIST trial suffers from significant drawbacks. One such limitation is the lack of differentiation between patients with simple and loculated empyema by CT or US scan that may be essential in selecting candidates for intrapleural fibrinolytics. A focus on patients with complicated parapneumonic effusions, rather than the current studies on heterogenous groups could clarify the specific area in which fibrinolytics may play a role [24].

\section{Thoracoscopy}

Thoracoscopy has been used as an alternative to thoracotomy in pleural effusion due to lung infection, because it allows the mechanical removal of infected material and permits lung re-expansion. It is possible to open multiple loculations and aspirate the purulent liquid, removing the fibrinous adhesions, including the layer on visceral pleura. Therefore a single cavity can be obtained in which, using an accurately positioned chest tube, subsequent local treatment is facilitated with antiseptic solutions or fibrinolytics. Moreover, the possibility of performing pleural biopsies allows the precise aetiological definition of the disease and aids the diagnosis of occult infections (e.g. tuberculosis) or tumours causing pleural effusion [25].

Most thoracoscopic empyema treatments are performed and described by surgeons using classical three-entry port intervention under general anaesthesia and double-lumen intubation (VATS) but thoracoscopic procedures also include medical thoracoscopy (MT). 
MT can be performed by a pulmonologist in an endoscopy suite using local anaesthetic and moderate sedation or general anaesthesia without intubation.

US identifies an entry site for the thoracoscope where the effusion is largest and furthest from the diaphragm. The advantages of medical thoracoscopy compared with VATS include lower cost and better tolerance by frail patients who may not tolerate general anaesthesia with tracheal intubation, which is required for VATS.

In patients with multiloculated thoracic empyema stratified by ultrasonography and treated early with medical thoracoscopy, a success rate of 93\% was reported with a small proportion of patients needing conversion to VATS or open surgery [15] (figure 2).

The exact application of thoracoscopy in infections of the pleural cavity has yet to be established. Its use has been proposed prior to the positioning of a thoracic drain, while another application might be proposed only after the drain has not been successful in determining a reduction in temperature or the complete evacuation of pleural fluid within a few days. Yet another approach makes reference to the loculate character of the effusion, and considers thoracoscopy appropriate in the presence of loculated empyema. More recently in the surgical field, the treatment has been extended to chronic organising empyema, both to clean the cavity prior to thoracotomic decortication, and for the actual decortications [25].

The studies on the role of thoracoscopy in the infection of the pleural space deal principally with empyema and are both medical and surgical, the latter being the subject of more studies.

VATS in patients with fibrinopurulent effusion demonstrate favourable results, with percentages of primary success (meaning complete cure without subsequent thoracotomy or conversion from VATS to thoracotomy) between $60 \%$ and $100 \%$, and higher if the method was used without delay.

However the number of patients treated was generally small, and few authors present case studies dealing with more than 50 patients [27].

In the surgical field there is unanimous agreement regarding the advantages of VATS over tho- racotomy, in terms of lower cost, shorter hospitalisation, and better cosmetic results. There is, however, less agreement about the advantages of MT over VATS in empyema, despite the former's mini invasiveness, lower cost and its advantages in patients with high surgical risk.

\section{Surgical decortication}

Decortication is a major thoracic operation, frequently requiring a thoracotomy incision. It is still indicated in cases when, 6 months after the acute stage, the pleura is still thickened and the patient's pulmonary function is sufficiently reduced to limit normal activities [9].

\section{Conclusion}

Pleural empyema is undoubtedly a complex issue in which some aspects have been clarified, such as the role of imaging techniques, the function of diagnostic thoracentesis and pleural fluid analysis, and the application of antibiotic therapy. There are, however, many aspects still under discussion, such as the use of fibrinolytics, and the indications of drainage techniques (chest tube insertion and thoracoscopy) and timing of their application.

This is true, in particular, for thoracoscopy, surgical (VATS) [27] or medical (MT) [12], which is certainly effective, especially in multiloculated empyema, allowing treatment without thoracotomy, even if large randomised studies on it have yet to be performed [25].

Medical thoracoscopy can play an important role, in particular for patients in poor health at high surgical risk. Results of recent studies on patients with multiloculated thoracic empyema stratified by US and treated early with MT showed that this approach is safe, minimally invasive, and efficient [15], provided that it is performed rapidly to avoid chronic evolution of the disease. Further trials on its application in empyema are still required in order to provide the basis for more substantial conclusions.

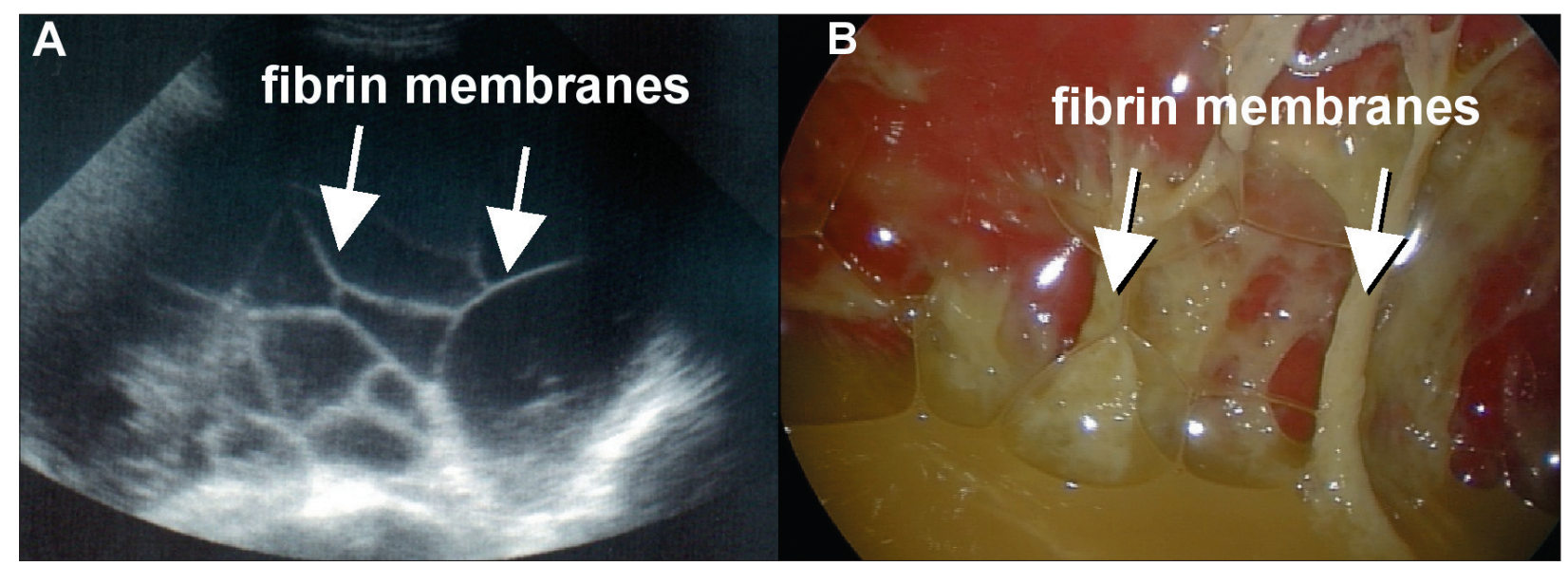

Fig. 2. - Sonographic (A) and thoracoscopic appearance (B) of a complex septated parapneumonic effusion with fibrin membranes in the pleural cavity. 
Acknowledgements: We would like to thank Mr. Robert Ellis for his help in checking the English language.

\section{References}

1. Aelony Y. Parapneumonic effusion does not equal empyema. Chest 1998; 114: 658-9.

2. Tassi GF, Marchetti GP. Pleural disease: historic perspective. In: Light RW, Gary Lee YC, eds. Textbook of pleural diseases, $2^{\text {nd }}$ edition. London: Hodder Arnold, 2008: 1-9.

3. Maskell NA, Batt S, Hedley EL, et al. The bacteriology of pleural infection by genetic and standard methods and its mortality significance. Am J Respir Crit Care Med 2006; 174: 817-824.

4. Colice GL, Curtis A, Deslauriers J, et al. Medical and surgical treatment of parapneumonic effusions: an evidence-based guideline. Chest 2000; 118: 1158-1171.

5. Farjah F, Symons RG, Krishnadasan B, et al. Management of pleural space infections: a population-based analysis. J Thorac Cardiovasc Surg 2007; 133: 346-51.

6. Finley C, Clifton J, FitzGerald JM, et al. Empyema: an increasing concern in Canada. Can Respir J 2008; 15 : 85-89.

7. Davies HE, Davies RJO, Davies CWH. Management of pleural infection in adults: British Thoracic Society pleural disease guideline 2010. Thorax 2010; 65: ii41-ii53.

8. Colice GL, Curtis A, Deslauriers J, et al. Medical and surgical treatment of parapneumonic effusions: an evidence-based guideline. Chest 2000; 118: 1158-1171.

9. Light RW. Parapneumonic Effusions and Empyema. Proc Am Thorac Soc 2006; 3: 75-80.

10. Andrews NC, Parker EF, Shaw RP, et al. Management of nontuberculous empyema. A statement of the subcommittee on surgery. Am Rev Respir Dis 1962; 85: 935-936.

11. Heffner JE, Klein S, Hampson C. Diagnostic utility and clinical application of imaging for pleural space infections. Chest 2010; 137: 467-79.

12. Tassi GF, Davies RJ, Noppen M. Advanced techniques in medical thoracoscopy. Eur Respir J 2006; 28: 10511059.

13. Chen KY, Liaw YS, Wang HC, et al. Sonographic septation: a useful prognostic indicator of acute thoracic empyema. J Ultrasound Med 2000; 19: 837-43.
14. Chiu CY, Wong KS, Huang YC, et al. Echo-guided management of complicated parapneumonic effusion in children. Pediatr Pulmonol 2006; 41: 1226-32.

15. Brutsche M H., Tassi GF, Györik S, et al. Treatment of sonographically statified multiloculated thoracic empiema by medical thoracoscopy. Chest 2005; 128: 3303 3309.

16. Froudarakis ME. Diagnostic work-up of pleural effusion. Respiration 2008; 75: 4-13.

17. Mishra EK, Rahman NM. Factors influencing the measurement of pleural fluid pH. Curr Opin Pulm Med 2009; 15: 353-7.

18. Jimènez D, Diaz G, Garcia-Rull S, et al. Routine use of pleural fluid cultures. Are they indicated? Limited yield, minimal impact on treatment decisions. Respir Med 2006; 100: 2048-52.

19. Heffner JE, Klein JS, Hampson C. Interventional management of pleural infections. Chest 2009; 136: 1148-59.

20. Rhaman NM, Maskell NA, Davies CW, et al. The relationship between chest tube size and clinical outcome in pleural infection. Chest 2010; 137: 536-43.

21. Davies HE, Rahman NM, Parker RJ, et al. Use of indwelling pleural catheters for chronic pleural infection. Chest 2008; 133: 546-9.

22. Maskell NA, Davies CW, Nunn AJ, et al. First Multicenter Intrapleural Sepsis Trial (MIST1) Group U.K. Controlled trial of intrapleural streptokinase for pleural infection. N Engl J Med 2005; 352: 865-874.

23. Misthos P, Sepsas E, Konstantinou M, et al. Early use of intrapleural fibrinolytics in the management of postpneumonic empyema. A prospective study. Eur $J$ Cardiothorac Surg 2005; 28: 599-603.

24. Cameron R, Davies HR. Intra-pleural fibrinolytic therapy versus conservative management in the treatment of adult parapneumonic effusions and empyema. Cochrane Database Syst Rev. 2008; 2: CD002312.

25. Waller DA. Thoracoscopy in management of postpneumonic pleural infections. Curr Opin Pulm Med 2008; 4 : 323-326.

26. Tassi GF, Davies RJ, Noppen M. Advanced techniques in medical thoracoscopy. Eur Respir J 2006; 28: 10511059.

27. Luh SP, Chou MC, Wang LS, et al. Video-assisted thoracoscopic surgery in the treatment of complicated parapneumonic effusions or empyemas: outcome of 234 patients. Chest 2005; 127: 1427-1432.

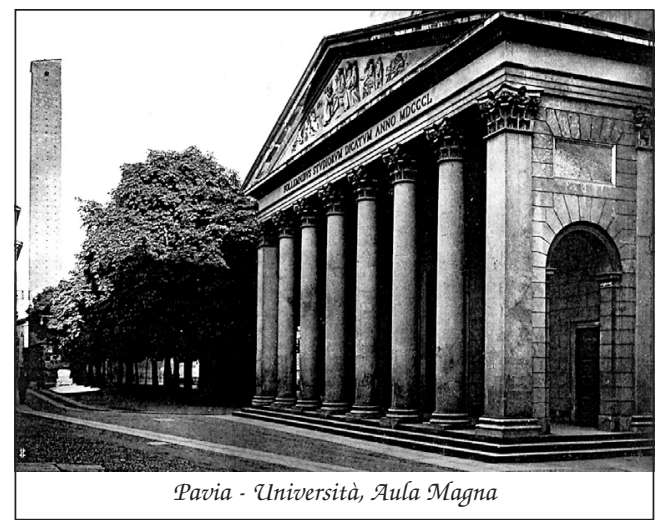

